\title{
Seizures arising from the inferior parietal lobule can show ictal semiology of the second sensory seizure (SII seizure)
}

\author{
J Yamamoto, A Ikeda, M Matsuhashi, T Satow, M Takayama, S Ohara, R Matsumoto, \\ N Mikuni, J Takahashi, S Miyamoto, W Taki, N Hashimoto, H Shibasaki
}

J Neurol Neurosurg Psychiatry 2003;74:367-369

\begin{abstract}
A 52 year old right handed man presented with medically intractable partial seizures consisting of numbness on the left upper back spreading to the left upper as well as lower limbs. Head computed tomography and magnetic resonance imaging showed a round calcified lesion in the depth of the superior ramus of the right sylvian fissure. Ictal electrocorticographic recording with chronically implanted subdural electrodes showed low voltage fast activities starting exclusively from an electrode located on the right inferior parietal lobule. No apparent ictal activities were observed from the depth electrodes inserted in the parietal operculum. Somatosensory evoked potentials of $75 \mathrm{~ms}$ to $145 \mathrm{~ms}$ latency were recorded from the ictal onset zone, which was $2 \mathrm{~cm}$ caudal to the perisylvian area corresponding to the second somatosensory area. Seizures arising from the inferior parietal lobule including the angular and supuramarginal gyri can produce partial seizures whose ictal semiology and scalp electroencephalography are indistinguishable from the ones originating from the second somatosensory area.
\end{abstract}

$\mathrm{T}$ he ictal symptoms of the second somatosensory seizure (SII seizure) (seizures involving the second somatosensory area) by Penfield and Jasper ${ }^{1}$ are characterised by bilateral and/or more widespread, axial numbness, tingling or pain whose localisation in the body is different from the somatotopy of the primary sensory cortex,${ }^{1-6}$ and several similar cases have been reported. ${ }^{2-6}$ However, only a small number of those cases have been studied by intracranial recording. ${ }^{14}$ In humans, SII was rather indistinctly defined to be located in the parietal upper bank of the sylvian fissure, mainly based on the results of direct electric cortical stimulation. This notion has been supported by somatosensory evoked potential (SEP) findings, magnetoencephalographic studies, and more recently by neuroimaging studies. ${ }^{17-17}$ However, probably because of interindividual variability of the anatomical location of SII, and also because of a limited number of cases studied by intracranial recording, it is still unclear which part of the perisylvian cortex is responsible for the so called SII seizure. We evaluated a patient in whom the initial sensory symptoms were considered to be consistent with SII seizures and the ictal onset zone was finally defined in the parietotemporal perisylvian areas, clearly caudal to SII. Neurophysiological data of this patient were described elsewhere for an entirely different purpose (M Matsuhashi, et al, 8th international conference on functional mapping of the human brain, 2002. Abstract available on CD ROM).

\section{CASE REPORT}

A 52 year old right handed man was investigated because of medically intractable partial seizures. He began to have intractable partial seizures at the age of 20 years. The seizures always consisted of numbness at the left upper back that spread to the left upper extremity and the left hip, and then to the left lower extremity. This simple partial seizure (SPS) was sometimes followed by loss of consciousness and automatism involving the hands and feet. With various combinations of antiepileptic drugs including phenytoin, carbamazepine, and valproic acid, his complex partial seizures (CPSs) occurred with an average frequency of one to three times per month, the longest seizure free interval being one month and the maximum seizure frequency of one to two times a day.

On physical examination, he had no focal neurological abnormality. Brain compted tomography (CT) showed a round calcified lesion in the depth of the right sylvian fissure. FLAIR-MRI of the brain showed high intensity signal abnormality around the calcified lesion (fig 1A). Interictal ${ }^{18}$ FDG-PET showed a small area of hypometabolism around the calcified lesion.

The patient was studied by continuous video EEG monitoring for 13 days. Interictal spikes were infrequently observed at T6 and O2 (International 10-20 system). Although ictal EEGs recorded from the scalp electrodes did not clearly localise the seizure onset zone precisely, the ictal symptoms and the calcified lesion in the depth of the right sylvian fissure strongly suggested that the habitual seizures in this patient originated from the right parietal perisylvian area, especially from SII.

To further localise the epileptogenic and symptomatogenic areas before surgical treatment, the patient underwent implantation of two subdural grid electrodes $(2 \times 5)$, one each on the right frontal and temporal lobes (fig 1B), and one depth electrode in the right parietal operculum around the calcified lesion. Informed consent for this investigation was obtained from the patient (clinical protocol number 79 approved by the ethical committee of Kyoto University Graduate School of Medicine). The electrodes were $3 \mathrm{~mm}$ in diameter and placed with $1 \mathrm{~cm}$ interelectrode distance (centre to centre). The depth electrode had six contacts, each $2.3 \mathrm{~mm}$ of length and $1 \mathrm{~cm}$ of centre to centre distance (AD-TECH, Racine, WI, USA).

During invasive monitoring, the patient experienced more than one hundred SPSs and five CPSs. In all the recorded habitual seizures, low voltage fast activities of about $20 \mathrm{~Hz}$ started exclusively from electrode 14 (fig lC). In almost all the seizures, ictal activities started almost simultaneously with the clinical symptoms. Once ictal electrocortiographic activities propagated to the adjacent electrodes, the patient developed CPSs. No ictal activities were observed in the depth

Abbreviations: SEP; somatosensory evoked potiential; $\mathrm{CT}$, computed tomography; MRI, magnetic resonance imaging; Sll, second somatosensory area 


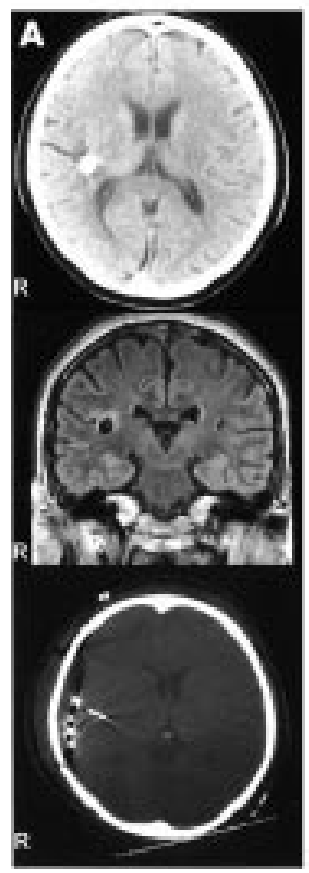

Seizure onset zone Face primary motor area
B

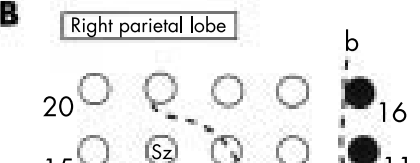

(a) Sylvian fissure

(b) Central sulcus

(c) Superior ramus of the sylvian fissure

(d) Inferior ramus of the sylvian fissure

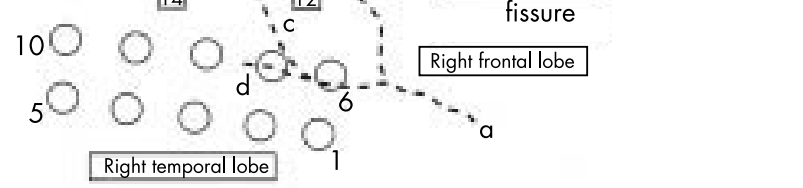

C

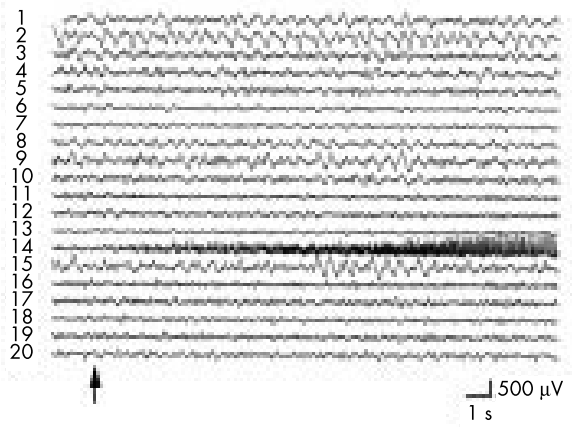

Figure 1 (A) Head CT (upper) and FLAIR MRI (middle) of the present patient before surgical resection. Head CT (lower) taken after the insertion of the depth electrodes. Upper: a round calcified mass is seen in the depth of the right sylvian fissure. Middle: the calcified mass is surrounded by high intensity signal abnormality. Lower: Three contacts of the depth electrodes were inserted from the parietal operculum to the calcified mass. Placement of subdural electrodes (B) and ictal

electrocorticographic records (C). Electrodes 11 and 16 correspond to the face area of the primary motor cortex as determined by electric stimulation. The seizure onset was always identified at electrode 14 Electrode 12 (SII) is located in the postcentral gyrus, and electrode 14 (seizure onset zone) is located in the inferior parietal lobule. The arrow indicated the onset of the clinical symptoms.

\begin{tabular}{|c|c|c|c|c|}
\hline & \multicolumn{2}{|l|}{ Electrode 14} & \multicolumn{2}{|l|}{ Electrode 12} \\
\hline & latency (ms) & amplitude $(\mathrm{mV})$ & latency (ms) & amplitude $(\mathrm{mV})$ \\
\hline $\begin{array}{l}\text { Left median nerve } \\
\text { Right median nerve } \\
\text { Left tibial nerve } \\
\text { Right tibial nerve }\end{array}$ & $\begin{array}{l}80 \\
80 \\
100 \\
100\end{array}$ & $\begin{array}{l}-30 \\
-30 \\
-30 \\
-30\end{array}$ & $\begin{array}{l}140 \\
140 \\
(-) \\
(-)\end{array}$ & $\begin{array}{l}35 \\
35 \\
(-) \\
(-)\end{array}$ \\
\hline
\end{tabular}

electrodes in the recorded four seizures, which included two habitual SPSs and two CPSs.

For functional mapping, high frequency electric cortical stimulation through subdural electrodes was used as previously described (SEN-7203 and ss-102J, Nihon-Koden, Japan). ${ }^{18}{ }^{19}$ Electric cortical stimulation between electrodes 11 and 16 produced contraction of the right oral angle. None of the other electrode pairs produced clinical symptoms even when stimulated with the maximum intensity of $15 \mathrm{~mA}$ (fig 1B).

For cortical SEP recording, median and tibial nerves were stimulated at the wrists and ankles, respectively, on each side separately with an electric pulse of $0.3 \mathrm{~ms}$ duration, and the intensity was adjusted just above the motor threshold (Pathfinder II, Nicolet Biomedical, WI, USA). All electrodes were referred to a scalp electrode placed on the skin over the mastoid process contralateral to the side of implantation. The interstimulus interval and the band pass were set to $3.3 \mathrm{~s}$ and 0.5-100 Hz, respectively. Cortical SEPs were identified from electrodes 12 and 14 with different latencies and somatotopic representations from each other (table 1). At electrode 14, cortical responses of $80-100 \mathrm{~ms}$ of latency were recognised after both the median and tibial nerve stimulation of either side, whereas at electrode 12, cortical responses of $140 \mathrm{~ms}$ were recorded only to the left and right median nerve stimulation.

Precise location of the electrode grids relative to the sylvian fissure as well as the central sulcus was confirmed by the observation during surgery and by $3 \mathrm{D}$ reconstructed MRI taken after implantation of the electrodes. Based on these findings, electrode 14 was situated just below the superior ramus of the sylvian fissure in the inferior parietal lobule where the supramarginal and angular gyri were located. Electrode 12 was located on the postcentral gyrus.

The patient underwent lesionectomy and resection of the cortical area covered by electrodes 13, 14, and 15. Histologically the calcified lesion was a psammomatous meningioma and its surrounding tissue was cortical dysplasia. The patient had been free of seizure for one year after the resection, and afterwards he gradually started having simple partial seizure consisting of numbness at the left upper back again, but with lesser frequency as compared with the presurgical state.

\section{DISCUSSION}

In this patient, the ictal symptoms consisting of numbness on the left upper back rapidly spreading to the left hand and foot and the existence of a calcified lesion in the depth of the right sylvian fissure (fig lA) strongly suggested the right SII as the epileptogenic or symptomatogenic zone.

We recorded more than 100 habitual seizures during the invasive monitoring. In most of those seizures, ictal activities started almost simultaneously with the ictal symptoms. Therefore, the area underlying electrode 14 was considered to be both the ictal onset and symptomatogenic zone, and thus electrode 14 was thought to be at or close to SII. However, electrode 14 was located at the area just below the superior ramus of the sylvian fissure that corresponded to the inferior 
parietal lobule including the supramarginal and angular gyri anatomically. ${ }^{20}$

Although human SII has been defined to be located in the parietal upper bank of the sylvian fissure by direct electric cortical stimulation, SEP $^{17-11}$ and somatosensory evoked magnetic field (SEF), ${ }^{12-14}$ its precise anatomical location is still poorly defined. Moreover, only a few cases of SII seizures were studied by intracranial recordings, ${ }^{14}$ and none of them described anatomical location of the ictal onset zone in detail. Therefore, the responsible area for the so called SII seizure may actually include not only SII itself but also the adjacent cortical area, such as the angular and supramarginal gyri.

From electrodes 12 and 14, cortical SEPs of different latencies and somatotopic representations were recorded. Long latency (80-140 ms) and bilateral representation (table 1) are characteristic features of SEPs arising from SII. ${ }^{10-14}$ At electrode 12, however, only median nerve electric stimulation produced clear, long latency SEPs, while at electrode 14 prominent SEPs were recorded after both median and tibial nerve stimulation. It may suggest that SEPs at electrode 12 reflect the somatotopic organization of SII as described in the previous reports, which showed that the hand area was most laterally and the foot area was most medially located along the upper bank of the sylvian fissure and the parietal operculum. ${ }^{12-14}$ Thus based on the present findings of cortical SEPs and anatomical data, electrode 12 is considered to be at or very close to SII.

Electrode 14 is outside the conventionally accepted SII region. However, the generation of long latency SEPs in this area after bilateral median and tibial nerve stimulation indicate that this particular area also plays a certain part in the sensory processing different from that at electrode 12 (M Matsuhashi, et al, 8th international conference on functional mapping of the human brain, 2002). According to the primate studies, area $7 \mathrm{~b}$, the retroinsular and posterior auditory areas, and the granular portion of the insula may account for the bilateral and/or diffusely contralateral sensory symptoms. ${ }^{22} 23$ Therefore, the area covered by electrode 14 could be responsible for the generation of partial seizures whose ictal semiology is indistinguishable from that of SII origin.

\section{ACKNOWLEDGEMENT}

This study was supported by Grants-in-Aid for Scientific Research on Priority area (C) (Advanced Brain Science) 12210012, Scientific Research (B2) 13470134 (C2) 13670640 from Japan Ministry of Education, Culture, Sports, Science and Technology. Research for the Future Program JSPS-RFTF97L00201 form the Japan Society for the Promotion of Science, and the Research Grants from the Japan Epilepsy Research Foundation, Brain Science Foundation and Magnetic Health Science Foundation.

\section{Authors' affiliations}

J Yamamoto, M Matsuhashi, T Satow, M Takayama, S Ohara, R Matsumoto, H Shibasaki, Human Brain Research Centre, Kyoto

University, Graduate School of Medicine, Kyoto, Japan

A lkeda, H Shibasaki, Department of Neurology, Kyoto University, Graduate School of Medicine

T Satow, M Takayama, N Mikuni, J Takahashi, S Miyamoto, N Hashimoto, Department of Neurosurgery, Kyoto University, Graduate School of Medicine

J Yamamoto, W Taki, Department of Neurosurgery, Mie University
School of Medicine, Mie, Japan

Competing interests: none declared.

Correspondence to: Dr A Ikeda, Department of Neurology, Kyoto University Graduate School of Medicine, Shogoin, Sakyo, Kyoto 606-8507, Japan; akio@kuhp.kyoto-u.ac.jp

Received 27 May 2002

Accepted in revised form 20 November 2002

\section{REFERENCES}

1 Penfield W, Jasper H. Epilepsy and the functional anatomy of the human brain. Boston, MA: Little, Brown, 1954

2 Scholz J, Vieregge P, Moser A. Central pain as a manifestation of partial epileptic seizures. Pain 1999;80:445-50

3 Otani K, Imai K, Futagi Y, et al. Bilateral painful epileptic seizures of the hands. Dev Med Child Neurol 1995;37:933-6.

4 Blume WT, Jones DC, Young GB, et al. Seizures involving secondary sensory and related areas. Brain 1992;115:1509-20.

5 Young GB, Barr HW, Blume WT. Painful epileptic seizures involving the second sensory area. [Letter]. Ann Neurol 1986;19:412.

6 Lesser RP, Lüders $\mathrm{H}$, Conomy JP, et al. Sensory seizure mimicking a psychogenic seizure. Neurology 1983;33:800-2.

7 Penfield W, Rasmussen T. Cerebral cortex of man: a clinical study of localization of function. New York: Macmillan, 1950

8 Woolsey CN, Erickson TC, Gilson WE. Localization in somatic sensory and motor areas of human cerebral cortex as determined by direct recording of evoked potentials and electrical stimulation. J Neurosurg 1979;51:476-506.

9 Lüders H, Lesser RP, Dinner DS, et al. The second sensory area in humans: evoked potential and electrical stimulation studies. Ann Neurol 1985; 17: 177-84.

10 Allison T, McCarthy G, Wood CC, et al. Human cortical potentials evoked by stimulation of the median nerve. II. Cytoarchitectonic areas generating long-latency activity. J Neurophysiol 1989;62:71 1-22.

11 Mima T, Ikeda A, Nagamine T, et al. Human second somatosensory area: subdural and magnetoencephalographic recording of somatosensory evoked reponses. J Neurol Neurosurg Psychiatry 1997;63:501-5.

12 Hari $\mathbf{R}$, Hämäläinen $M$, Kaukoranta $E$, et al. Neuromagnetic responses from the second somatosensory cortex in man. Acta Neurol Scand 1984; 57:254-63.

13 Hari R, Karhu J, Hämäläinen $M$, et al. Functional organization of the human first and second somatosensory cortices: a neuromagnetic study. Eur J Neurosci 1993;5:724-34.

14 Maeda K, Kakigi R, Hoshiyama M, et al. Topography of the second somatosensory cortex in humans: a magnetoencephalographic study. Neuroreport 1999; 10:301-6.

15 Burton H, Videen TO, Raichle ME. Tactile-vibration-activated foci in insular and parietal-opercular cortex studied with positron emission tomography: mapping the second somatosensory area in humans. Somatosens Motor Res 1993;10:297-308.

16 Disbrow E, Roberts T, Krubitzer L. Somatotopic organization of cortical fields in the lateral sulcus of Homo sapiens: evidence for SII and PV. J Comp Neurol 2000;418:1-21

17 Ruben J, Schwiemann J, Deuchert M, et al. Somatotopic organization of human secondary somatosensory cortex. Cereb Cortex 2001:11:463-73.

18 Lüders HO, Lesser RP, Dinner DS, et al. Commentary: Chronic intracranial recording and stimulation with subdural electrodes. In: Enge $\mathrm{Jr}$, eds. Surgical treatment of the epilepsies. New York: Raven Press, 1987:297-321.

19 Ikeda A, Lüders HO, Burgess RC, et al. Movement-related potentials recorded from supplementary motor area and primary motor area: role of supplementary motor area in voluntary movements. Brain 1992; 115:1017-43.

20 Parent A. Carpenter's human neuroanatomy. Regional anatomy of the brain. 9th edn. Pennsylvania: Williams and Willkins, 1996.

21 Robinson CJ, Burton H. Somatotopographic organization in the second somatosensory area of M fascicularis. J Comp Neurol 1980;192:43-67.

22 Robinson CJ, Burton H. Organization of somatosensory receptive fields in cortical areas $7 b$, retroinsula, postauditory and granular insula of $M$ fascicularis. J Comp Neurol 1980;192:69-92.

23 Robinson CJ, Burton H. Somatic submodality distribution within the second somatosensory (SII), 7b, retroinsular, postauditory, and granular insular cortical areas of $M$ fascicularis. J Comp Neurol 1980;192:93-108. 